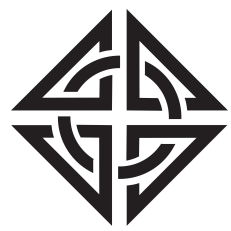

SCIENTIA

I RAN I C A
Sharif University of Technology

Scientia Iranica

Transactions B: Mechanical Engineering

www.scientiairanica.com

\title{
Sub-equation method for the fractional regularized long-wave equations with conformable fractional derivatives
}

\author{
H. Aminikhah ${ }^{a, *}$, A.H. Refahi Sheikhani ${ }^{b}$ and H. Rezazadeh ${ }^{a}$ \\ a. Department of Applied Mathematics, Faculty of Mathematical Sciences, University of Guilan, Rasht, P.O. Box 1914, Iran. \\ b. Department of Applied Mathematics, Islamic Azad University, Lahijan Branch, Lahijan, P.O. Box 1616, Iran.
}

Received 6 February 2015; received in revised form 6 July 2015; accepted 8 August 2015

\section{KEYWORDS}

Sub-equation method;

Conformable

fractional derivative;

Regularized long-wave

equation;

Exact solutions.

\begin{abstract}
In this paper, we employ a sub-equation method to find the exact solutions to the fractional $(1+1)$ and $(2+1)$ regularized long-wave equations which arise in several physical applications, including ion sound waves in plasma, by using a new definition of fractional derivative called conformable fractional derivative. The presented method is more effective, powerful, and straightforward and can be used for many other nonlinear partial fractional differential equations.
\end{abstract}

(C) 2016 Sharif University of Technology. All rights reserved.

\section{Introduction}

In recent years, fractional differential equations are increasingly utilized to model many problems in biology, chemistry, engineering, physics, economic, and other areas of applications [1-5]. The fractional differential equations have become a useful tool for describing nonlinear phenomena of science and engineering models. There are several definitions for fractional differential equations. The most popular definitions in the world of fractional calculus are [6]:

(i) The Riemann-Liouville derivative of fractional order of function, given as:

$$
\begin{aligned}
D_{t}^{\alpha} f(t) & =\frac{1}{\Gamma(1-\alpha)} \frac{d}{d t} \int_{0}^{t} \frac{f(\varepsilon)}{(t-\varepsilon)^{\alpha}} d(\varepsilon), \\
0 & <\alpha \leq 1
\end{aligned}
$$

*. Corresponding author. Tel.:+98 1333362208

E-mail addresses: hossein.aminikhah@gmail.com, aminikhah@guilan.ac.ir (H.Aminikhah);

ah_refahi@yahoo.com,ah_refahi@guilan,ac.ir (A. Refahi

Sheikhani); rezazadehadi1363@gmail.com (H. Rezazadeh). (ii) The Caputo fractional derivative of a differentiable function says $f(t)$ is defined as:

$$
\begin{aligned}
D_{t}^{\alpha} f(t) & =\frac{1}{\Gamma(1-\alpha)} \int_{0}^{t} \frac{f^{\prime}(\varepsilon)}{(t-\varepsilon)^{\alpha}} d x \\
0 & <\alpha \leq 1
\end{aligned}
$$

Furthermore, Jumarie in [7] proposed the following modified Riemann-Liouville fractional derivative:

$$
\begin{aligned}
D_{t}^{\alpha} f(t) & =\frac{1}{\Gamma(1-\alpha)} \frac{d}{d t} \int_{0}^{t}(t-\varepsilon)^{-\alpha}(f(\varepsilon)-f(0)) d \varepsilon \\
0 & <\alpha \leq 1
\end{aligned}
$$

We give some basic properties for the modified Riemann-Liouville derivative as follows (see [7, formulae (3.12) and (3.13)]):

$$
\begin{aligned}
& D_{x}^{\alpha}(u(x) v(x))=v(x) D_{x}^{\alpha}(u(x))+u(x) D_{x}^{\alpha}(v(x)), \\
& D_{x}^{\alpha}[f(u(x))]=D_{x}^{\alpha} f(u)\left(u_{x}^{\prime}\right)^{\alpha}, \\
& D_{x}^{\alpha}[f(u(x))]=f_{u}^{\prime}(u) D_{x}^{\alpha} u(x) .
\end{aligned}
$$


During the last few years, the exact solution methods have been proposed for solving fractional differential equations, e.g. the functional variable method [8], the first integral method [9], the exp-function method [10], fractional Riccati expansion method [11-12], and so on [13-14]. Eqs. (5) and (6) are applied in many papers to solve the exact solutions to some nonlinear fractional differential equations; if Eq. (6) is correct, then using the variable transformation $\xi=\frac{k x+l y+c t^{\alpha}}{\Gamma(\alpha+1)}$, where $k, l$, and $c$ are constants, the fractional partial derivative $\frac{\partial^{\alpha} u(x, y, t)}{\partial t^{\alpha}}$ is reduced to ordinary derivative $c u^{\prime}(\xi)$. Therefore, the corresponding fractional differential equations become the ordinary differential equations which are easy to study. But Liu in [15] has shown that Jumarie's basic (Eqs. (4), (5), and (6)) are incorrect; thus, the corresponding results on differential equations are not correct.

Recently, to dominate these and other difficulties, Khalil et al. [16] introduced a new simple wellbehaved definition of the fractional derivative called conformable fractional derivative. This fractional derivative is theoretically very easier to handle and also obeys some conventional properties that cannot be satisfied by the existing fractional derivatives, for instance, the chain rule [17]. The conformable fractional derivative has the weakness that the fractional derivative of any differentiable function at the point zero is equal to zero. Atangana et al. [18-20] proposed a suitable fractional derivative that allows us to escape the lack of the conformable fractional derivative. The $\beta$-derivative of a function $f:[\alpha, \infty] \rightarrow \mathbb{R}$ is defined as:

$$
{ }_{0}^{A} D_{t}^{\beta}(f(t))=\lim _{\varepsilon \rightarrow 0} \frac{f\left(t+\varepsilon\left(t+\frac{1}{\Gamma(\beta)}\right)^{1-\beta}\right)-f(t)}{\varepsilon},
$$

for all $t \geq \alpha, 0<\beta \leq 1$. Then, if the above limit exists, $f$ is said to be $\beta$-differentiable.

In this paper, we obtain the exact solutions to the one- and two-dimensional fractional Regularized Long-Wave (RLW) equations by means of conformable fractional derivative by using the sub-equation method. The sub-equation method is a powerful solution method for the computation of exact traveling wave solutions. This method is one of the most direct and effective algebraic methods for finding exact solutions to nonlinear Fractional Partial Differential Equations (FPDEs).

\section{Conformable fractional derivative}

Grunwald-Letnikov, Riemann-Liouville, Caputo, Weyl, Marchaud, and Riesz definitions of fractional derivatives attempt to satisfy the usual properties of the standard derivative. The only property inherited by all the definitions of fractional derivatives is the linearity property. Some properties of one definition or another are listed as follows $[6,16]$ :

(i) Most of the fractional derivatives do not satisfy $D_{t}^{\alpha}(C)=0$ (C is a constant $)$, if $\alpha$ is not a natural number.

(ii) All fractional derivatives do not obey the known product rule for two functions:

$$
D_{t}^{\alpha}(f g)=f D_{t}^{\alpha}(g)+g D_{t}^{\alpha}(f) .
$$

(iii) All fractional derivatives do not satisfy the known quotient rule for two functions:

$$
D_{t}^{\alpha}\left(\frac{f}{g}\right)=\frac{f D_{t}^{\alpha}(g)-g D_{t}^{\alpha}(f)}{g^{2}} .
$$

(iv) All fractional derivatives do not obey the chain rule:

$$
D_{t}^{\alpha}(f \circ g)(t)=D_{t}^{\alpha} f(g(t)) D_{t}^{\alpha} g(t) .
$$

(v) All fractional derivatives do not satisfy $D_{t}^{\alpha} D_{t}^{\beta} f=$ $D_{t}^{\alpha+\beta} f$, in general.

(vi) Caputo definition assumes that the function $f$ is differentiable.

Recently, Khalil et al. in [16] introduced a new definition of fractional derivative to overcome some of these and other difficulties, as follows:

Definition 1 [16]. Let $f:(0, \infty) \rightarrow \mathbb{R}$, then, the conformable fractional derivative of $f$ of order $\alpha$ is defined as:

$$
{ }_{t} T_{\alpha} f(t)=\lim _{\varepsilon \rightarrow 0} \frac{f\left(t+\varepsilon t^{1-\alpha}\right)-f(t)}{\varepsilon},
$$

for all $t>0,0<\alpha \leq 1$. If $f$ is $\alpha$-differentiable in some $(0, a), a>0$, and $\lim _{t \rightarrow 0^{+}}{ }_{t} T_{\alpha} f(t)$ exists, then, by definition:

$$
{ }_{t} T_{\alpha} f(0)=\lim _{t \rightarrow 0^{+}}{ }_{t} T_{\alpha} f(t) .
$$

In [16], authors have proved that the new fractional derivative satisfies the product rule (Eq. (8)) and Quotient rule (Eq. (9)). If, in addition, $f$ is differentiable, then, ${ }_{t} T_{\alpha}(f)(t)=t^{1-\alpha} \frac{d f}{d t}$.

In [17], T. Abdeljawad established the chain rule for conformable fractional derivatives as the following theorem:

Theorem 1 (chain rule). Let $f, g:(0, \infty) \rightarrow \mathbb{R}$ be $\alpha$-differentiable functions, where $0<\alpha \leq 1$. Then, $f(g(t))$ is $\alpha$-differentiable and for all $t$ with $t \neq 0$ and $g(t) \neq 0$, we have:

$$
{ }_{t} T_{\alpha}(f \circ g)(t)=\left({ }_{t} T_{\alpha} f\right)(g(t))\left({ }_{t} T_{\alpha} g\right)(t) g(t)^{\alpha-1} .
$$

If $t \neq 0$, then:

$$
{ }_{t} T_{\alpha}(f \circ g)(0)=\lim _{t \rightarrow 0^{+}}\left({ }_{t} T_{\alpha} f\right)(g(t))\left({ }_{t} T_{\alpha} g\right)(t) g(t)^{\alpha-1} .
$$


Theorem 2. Let $f:(0, \infty) \rightarrow \mathbb{R}$ be a function such that $f$ is differentiable and also $\alpha$-differentiable. Let $g$ be a function defined in the range of $f$ and also differentiable; then, one has the following rule:

$$
{ }_{t} T_{\alpha}(f \circ g)(t)=t^{1-\alpha} g^{\prime}(t) f^{\prime}(g(t)) .
$$

Now, we list here the fractional derivatives of certain functions:
(i) ${ }_{t} T_{\alpha}\left(e^{\frac{1}{\alpha} t^{\alpha}}\right)=e^{\frac{1}{\alpha} t^{\alpha}}$,
(ii) ${ }_{t} T_{\alpha}\left(\sin \frac{1}{\alpha} t^{\alpha}\right)=\cos \frac{1}{\alpha} t^{\alpha}$,
(iii) ${ }_{t} T_{\alpha}\left(\cos \frac{1}{\alpha} t^{\alpha}\right)=-\sin \frac{1}{\alpha} t^{\alpha}$,
(iv) ${ }_{t} T_{\alpha}\left(t^{\mu}\right)=\mu t^{\mu-\alpha}$, for all $\mu \in \mathbb{R}$.

On letting $\alpha=1$ in these derivatives, we get the corresponding ordinary derivatives.

Definition 2 (fractional integral) [16]. Let $a \geq 0$ and $t \geq a$. Also, let $f$ be a function defined on $(a, t)$ and $a \in \mathbb{R}$. Then, the $\alpha$-fractional integral of $f$ is defined by:

$$
{ }_{t} I_{a}^{\alpha} f(t)=\int_{a}^{t} \frac{f(x)}{x^{1-\alpha}} d x
$$

if the Riemann improper integral exists.

\section{The sub-equation method}

In this section, we describe the main steps of the sub-equation method for finding the exact solutions of Fractional Partial Differential Equations (FPDEs).

Remark 1. We write $\frac{\partial^{\alpha}}{\partial t^{\alpha}}(f)$ for ${ }_{t} T_{\alpha}(f)$ to denote the conformable fractional derivatives of $f$ with respect to the variable $t>0$ of order $\alpha$.

Suppose that a nonlinear fractional partial differential equation, say, in two or three independent variables, $x, y$, and $t$, is given by:

$$
\begin{aligned}
& F\left(u, \frac{\partial^{\alpha} u}{\partial t^{\alpha}}, \frac{\partial^{\alpha} u}{\partial x^{\alpha}}, \frac{\partial^{\alpha} u}{\partial y^{\alpha}}, \frac{\partial^{2 \alpha} u}{\partial t^{2 \alpha}}, \frac{\partial^{2 \alpha} u}{\partial t^{\alpha} \partial x^{\alpha}}, \ldots\right)=0, \\
& 0<\alpha \leq 1
\end{aligned}
$$

where $u(x, y, t)$ is an unknown function, $F$ is a polynomial in $u$ and its various partial derivatives, in which the highest order derivatives and nonlinear terms are involved.

The main steps of the sub-equation method [21] are summarized as follows:

Step 1. Using a wave transformation:

$$
u(x, y, t)=u(\xi), \quad \xi=k \frac{x^{\alpha}}{\alpha}+l \frac{y^{\alpha}}{\alpha}+c \frac{t^{\alpha}}{\alpha},
$$

where $k, l$, and $c$ are constants to be determined later.
This enables us to use the following changes:

$$
\begin{aligned}
\frac{\partial^{\alpha}}{\partial t^{\alpha}}(.) & =c \frac{d}{d \xi}(.), \frac{\partial^{\alpha}}{\partial x^{\alpha}}(.)=k \frac{d}{d \xi}(.), \frac{\partial^{\alpha}}{\partial y^{\alpha}}(.) \\
& =l \frac{d}{d \xi}, \frac{\partial^{2 \alpha}}{\partial t^{2 \alpha}}(.)=c^{2} \frac{d^{2}}{d \xi^{2}}, \ldots
\end{aligned}
$$

Then, Eq. (14) can be turned into the following fractional ordinary differential equation with respect to variable $\xi$ :

$$
G\left(u, u^{\prime}, u^{\prime \prime}, u^{\prime \prime \prime}, \ldots\right)=0
$$

where $G$ is a polynomial in $u$.

Step 2. We suppose that Eq. (16) has a solution in the form given below:

$$
u(\xi)=\sum_{i=0}^{n} a_{i} \varphi^{i},
$$

where $a_{i}(i=0,1,2, \ldots, n)$ are constants to be determined later with $a_{n} \neq 0$. The positive integer $n$ can be determined by considering the homogeneous balance between the highest order derivatives and nonlinear terms appearing in Eq. (16), and $\varphi=\varphi(\xi)$ satisfies the following Riccati equation:

$$
\varphi^{\prime}=\sigma+\varphi^{2},
$$

where $\sigma$ is a constant. By using Eq. (18) repeatedly, we can express all derivatives of $\varphi$ in terms of series in $\varphi$.It is found that the Riccati equation(Eq. (18)) admits several types of solutions [22]:

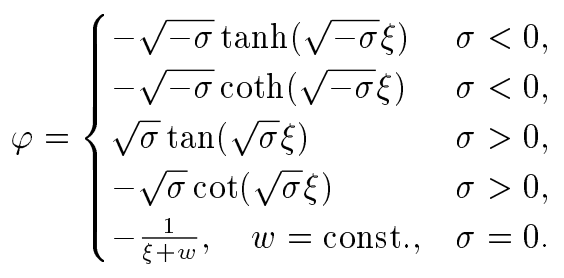

Step 3. Substituting Eqs. (17) and (18) into Eq. (16) and collecting the coefficients of $\varphi(\xi)$ and setting the coefficients of $[\varphi(\xi)]^{i}(i=0,1,2, \ldots)$ to be zero, we get an over-determined system of algebraic equations with respect to $a_{i}(i=0,1,2, \ldots, n)$ and $k, l, c$.

Step 4. Finally, assuming that $k, l, c$ and $a_{i}(i=$ $0,1,2, \ldots, n)$ are obtained by solving the algebraic equations in the previous step, and substituting these constants and the solutions of Eq. (18) into Eq. (17), we can obtain the explicit solutions of Eq. (16).

Remark 2. After applying this transformation to Eq. (14), by use of the chain rule (Eq. (13)), the original fractional partial differential equation can be transformed into another ordinary differential equation in one independent variable. 


\section{Application}

In this section, we construct the exact solutions for fractional RLW equations (Eqs. (20) and (21)) by using the sub-equation method described in Section 3.

One of the important nonlinear evolution equations by which we construct the exact solutions is the fractional Regularized Long-Wave equation (RLW). The fractional (1+1) RLW equation is as follows:

$$
\begin{gathered}
\frac{\partial^{\alpha}}{\partial t^{\alpha}} u+\frac{\partial^{\alpha}}{\partial x^{\alpha}} u+\varepsilon u \frac{\partial^{\alpha}}{\partial x^{\alpha}} u-\mu \frac{\partial^{3 \alpha}}{\partial t^{\alpha} \partial x^{2 \alpha}} u=0, \\
t>0, \quad 0<\alpha \leq 1
\end{gathered}
$$

where $\varepsilon$ and $\mu$ are positive constants and $u, t$, and $x$ denote the amplitude, time, and spatial coordinate, respectively. Moreover, the fractional $(2+1)$ RLW equation is as follows:

$$
\begin{gathered}
\frac{\partial^{\alpha}}{\partial t^{\alpha}} u+\frac{\partial^{\alpha}}{\partial x^{\alpha}} u+\frac{\partial^{\alpha}}{\partial y^{\alpha}} u+\varepsilon u \frac{\partial^{\alpha}}{\partial x^{\alpha}} u+\delta u \frac{\partial^{\alpha}}{\partial y^{\alpha}} u \\
-\mu \frac{\partial^{3 \alpha}}{\partial t^{\alpha} \partial x^{2 \alpha}} u-v \frac{\partial^{3 \alpha}}{\partial t^{\alpha} \partial y^{2 \alpha}} u=0 \\
t>0, \quad 0<\alpha \leq 1,
\end{gathered}
$$

where $\varepsilon, \delta, \mu$, and $v$ are positive constants and $u$, $t$ and $x, y$ denote the amplitude, time, and spatial coordinates, respectively. Further, $\alpha$ is a parameter describing the order of the fractional derivative. In the case of $\alpha=1$, the fractional RLW equation (one and two dimensional) decreases to the classical RLW equation. The RLW equations play a major role in the study of nonlinear dispersive waves since they describe a large number of important physical phenomena, such as shallow water waves and ion-acoustic plasma waves $[23,24]$.

\subsection{The one-dimensional fractional $R L W$ equation}

Using a wave transformation:

$$
u(x, t)=u(\xi), \quad \xi=k \frac{x^{\alpha}}{a} c \frac{t^{\alpha}}{\alpha} .
$$

Eq. (20) can be reduced to the following nonlinear ordinary differential equation:

$$
(c+k) u^{\prime}+k \varepsilon u u^{\prime}-k^{2} c \mu u^{\prime \prime \prime}=0 .
$$

We suppose that Eq. (22) has a solution in the form given below:

$$
u(\xi)=\sum_{i=0}^{n} a_{i} \varphi^{i},
$$

where $a_{i}(i=1,2, \ldots, n)$ are constants. Balancing the highest order derivative term $u^{\prime \prime \prime}$ and with nonlinear term $u u^{\prime}$ in Eq. (22), we can obtain $n=2$. So, we have:

$$
u(\xi)=a_{0}+a_{1} \varphi+a_{2} \varphi^{2} .
$$

Substituting Eq. (24) into Eq. (22), using Eq. (18), and collecting all the terms with the same power of $\varphi^{i}, i=$ $0,1, \ldots, 5$ equating each coefficient with zero, yields a set of algebraic equations. Solving these equations yields:

$$
a_{0}=-\frac{k+c-8 \mu k^{2} c \sigma}{\varepsilon k}, \quad a_{1}=0, \quad a_{2}=\frac{12 \mu k c}{\varepsilon} .
$$

Substituting the above result into Eq. (24) and combining it with Eq. (19), we obtain three types of exact solutions to Eq. (20), namely, two generalized hyperbolic function solutions, two generalized trigonometric function solutions, and one rational solution as follows:

$$
\begin{aligned}
u_{1}(x, t)= & -\frac{k+c-8 \mu k^{2} c \sigma}{\varepsilon k}-\frac{12 \mu k c \sigma}{\varepsilon} \\
& \tanh ^{2}\left(\sqrt{-\sigma}\left(k \frac{x^{\alpha}}{\alpha}+c \frac{t^{\alpha}}{\alpha}\right)\right), \quad \sigma<0, \\
u_{2}(x, t)= & -\frac{k+c-8 \mu k^{2} c \sigma}{\varepsilon k}-\frac{12 \mu k c \sigma}{\varepsilon} \\
& \operatorname{coth}^{2}\left(\sqrt{-\sigma}\left(k \frac{x^{\alpha}}{\alpha}+c \frac{t^{\alpha}}{\alpha}\right)\right), \quad \sigma<0, \\
u_{3}(x, t)= & -\frac{k+c-8 \mu k^{2} c \sigma}{\varepsilon k}+\frac{12 \mu k c \sigma}{\varepsilon} \\
& \tan ^{2}\left(\sqrt{\sigma}\left(k \frac{x^{\alpha}}{\alpha}+c \frac{t^{\alpha}}{\alpha}\right)\right), \quad \sigma>0, \\
u_{4}(x, t)= & -\frac{k+c-8 \mu k^{2} c \sigma}{\varepsilon k}+\frac{12 \mu k c \sigma}{\varepsilon} \\
& \cot ^{2}\left(\sqrt{\sigma}\left(k \frac{x^{\alpha}}{\alpha}+c \frac{t^{\alpha}}{\alpha}\right)\right), \quad \sigma>0, \\
u_{5}(x, t)= & -\frac{1}{\left(k \frac{x^{\alpha}}{\alpha}+c \frac{t^{\alpha}}{\alpha}\right)+w}, \\
w= & \operatorname{const}^{\sigma}=0 .
\end{aligned}
$$

In Figures 1 and 2, $u_{1}(x, t)$ shows one exact solution of Eq. (20) corresponding to the values $\alpha=1$ and $\alpha=0.9$ for $k=0.8, c=1.1, \sigma=-1.5, \mu=0.7$, and $\varepsilon=1.5$.

\subsection{The two-dimensional fractional $R L W$ equation}

Using a wave transformation:

$$
u(x, y, t)=u(\xi), \quad \xi=k \frac{x^{\alpha}}{\alpha}+l \frac{y^{\alpha}}{\alpha}+c \frac{t^{\alpha}}{\alpha} .
$$

Eq. (21) can be reduced to the following nonlinear 


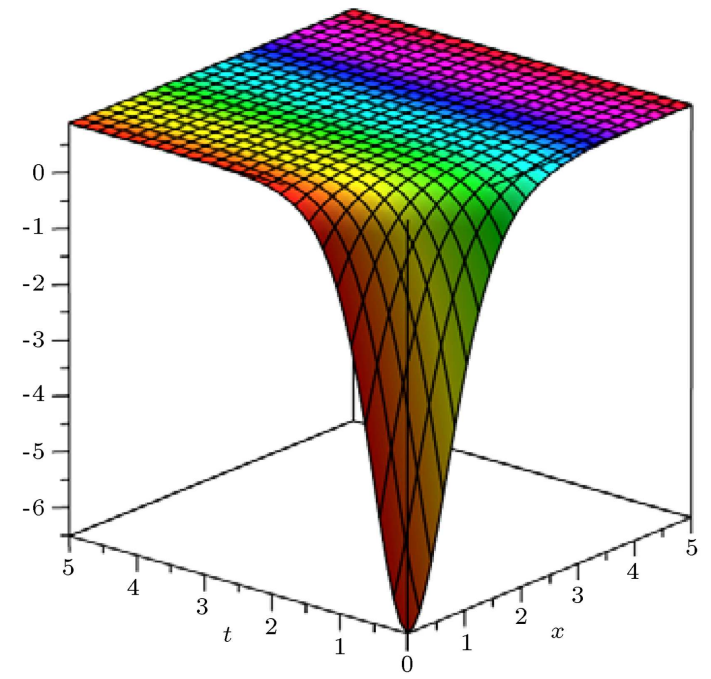

Figure 1. Graph of $u_{1}(x, t)$ of Eq. (20) corresponding to $\alpha=1$ for $k=0.8, c=1.1, \sigma=-1.5, \mu=0.7$, and $\varepsilon=1.5$.

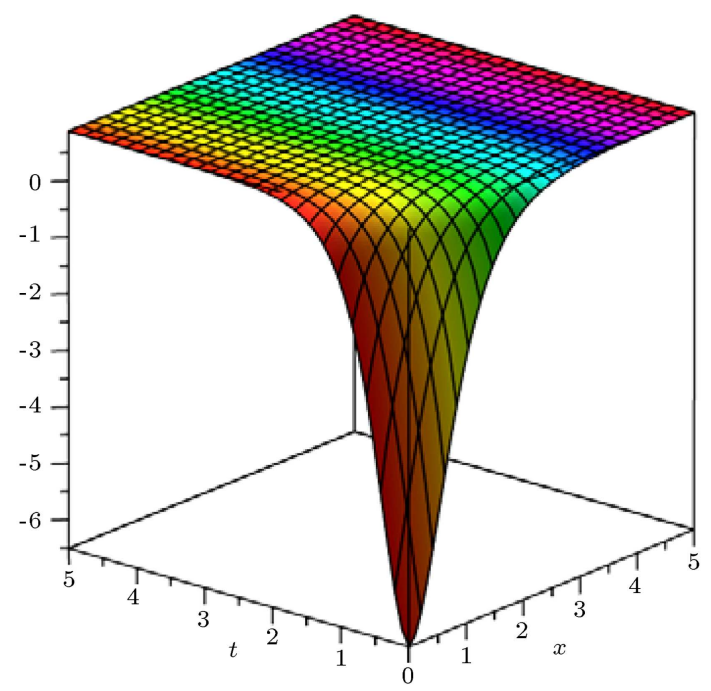

Figure 2. Graph of $u_{1}(x, t)$ of Eq. (20) corresponding to $\alpha=0.9$ for $k=0.8, c=1.1, \sigma=-1.5, \mu=0.7$, and $\varepsilon=1.5$.

fractional ordinary differential equation:

$$
(c+k+l) u^{\prime}+(\varepsilon k+\delta l) u u^{\prime}-c\left(\mu k^{2}+v l^{2}\right) u^{\prime \prime \prime}=0 .
$$

We suppose that Eq. (21) has a solution in the form given below:

$$
u(\xi)=\sum_{i=0}^{n} a_{i} \varphi^{i},
$$

where $a_{i}(i=0,1,2, \ldots, n)$ are constants. Balancing the highest order derivative term $u^{\prime \prime \prime}$ and with nonlinear term $u u^{\prime}$ in Eq. (25), we can obtain $n=2$. So, we have:

$$
u(\xi)=a_{0}+a_{1} \varphi+a_{2} \varphi^{2} .
$$

Substituting Eq. (27) into Eq.(25), using Eq. (18), and collecting all the terms with the same power of $\varphi^{i}(i=$ $0,1, \ldots, 5)$, equating each coefficient with zero, yields a set of algebraic equations. Solving these equations yields:

$$
\begin{aligned}
& a_{0}=-\frac{k+l+c-\left(8 \mu k^{2}+8 v l^{2}\right) c \sigma}{\varepsilon k+\delta k}, \\
& a_{1}=0, \quad a_{2}=\frac{12\left(\mu k^{2}+v l^{2}\right) c}{\varepsilon k+\delta l} .
\end{aligned}
$$

Substituting the above result into Eq. (25) and combining it with Eq. (19), we obtain three types of exact solutions to Eq. (21), namely, two generalized hyperbolic function solutions, two generalized trigonometric function solutions, and one rational solution as follows:

$$
\begin{aligned}
& u_{1}(x, y, t)=-\frac{k+l+c-\left(8 \mu k^{2}+8 v l^{2}\right) c \sigma}{\varepsilon k+\delta k} \\
& -\frac{12\left(\mu k^{2}+v l^{2}\right) c \sigma}{\varepsilon l+\delta l} \tanh ^{2}(\sqrt{-\sigma} \\
& \left.\left(k \frac{x^{\alpha}}{\alpha}+l \frac{y^{\alpha}}{\alpha}+c \frac{t^{\alpha}}{\alpha}\right)\right), \quad \sigma<0 \\
& u_{2}(x, y, t)=-\frac{k+l+c-\left(8 \mu k^{2}+8 v l^{2}\right) c \sigma}{\varepsilon k+\delta k} \\
& -\frac{12\left(\mu k^{2}+v l^{2}\right) c \sigma}{\varepsilon k+\delta l} \operatorname{coth}^{2}(\sqrt{-\sigma} \\
& \left.\left(k \frac{x^{\alpha}}{\alpha}+l \frac{y^{\alpha}}{\alpha}+c \frac{t^{\alpha}}{\alpha}\right)\right), \quad \sigma<0, \\
& u_{3}(x, y, t)=-\frac{k+l+c-\left(8 \mu k^{2}+8 v l^{2}\right) c \sigma}{\varepsilon k+\delta k} \\
& +\frac{12\left(\mu k^{2}+v l^{2}\right) c \sigma}{\varepsilon k+\delta l} \tan ^{2}(\sqrt{\sigma} \\
& \left.\left(k \frac{x^{\alpha}}{\alpha}+l \frac{y^{\alpha}}{\alpha}+c \frac{t^{\alpha}}{\alpha}\right)\right), \quad \sigma>0, \\
& u_{4}(x, y, t)=-\frac{k+l+c-\left(8 \mu k^{2}+8 v l^{2}\right) c \sigma}{\varepsilon k+\delta k} \\
& +\frac{12\left(\mu k^{2}+v l^{2}\right) c \sigma}{\varepsilon k+\delta l} \cot ^{2}(\sqrt{\sigma} \\
& \left.\left(k \frac{x^{\alpha}}{\alpha}+l \frac{y^{\alpha}}{\alpha}+c \frac{t^{\alpha}}{\alpha}\right)\right), \quad \sigma>0, \\
& u_{5}(x, y, t)=-\frac{1}{\left(k \frac{x^{\alpha}}{\alpha}+l \frac{y^{\alpha}}{\alpha}+c \frac{t^{\alpha}}{\alpha}\right)+w}, \\
& w=\text { const. }, \quad \sigma=0 .
\end{aligned}
$$

In Figures 3 and $4, u_{4}(x, y, t)$ shows one exact solution 


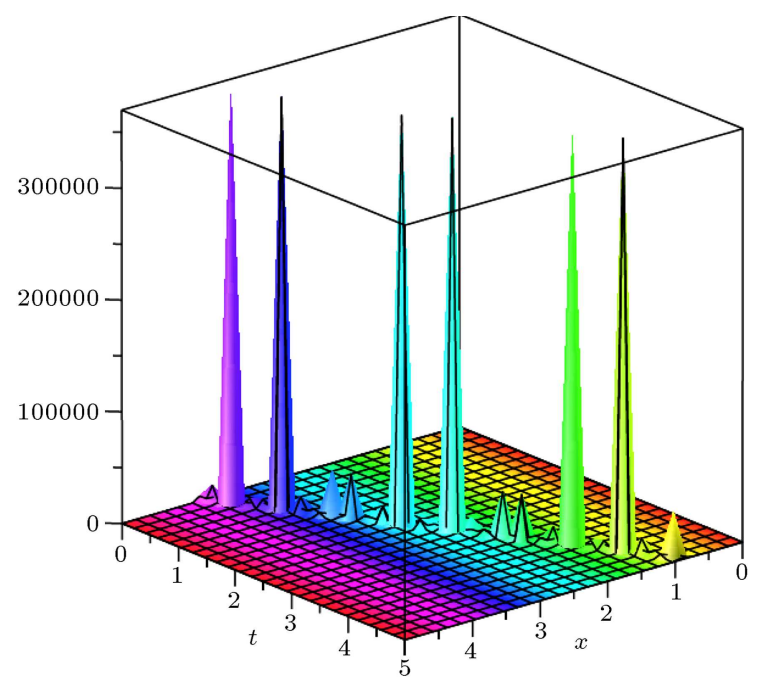

Figure 3. Graph of $u_{4}(x, y, t)$ of Eq. (21) corresponding to $\alpha=1$ for $k=0.85, c=0.5, l=0.5, \sigma=0.75, \mu=0.8$, $\delta=1.5, v=1.2$, and $\varepsilon=1.6$ at $y=0.75$.

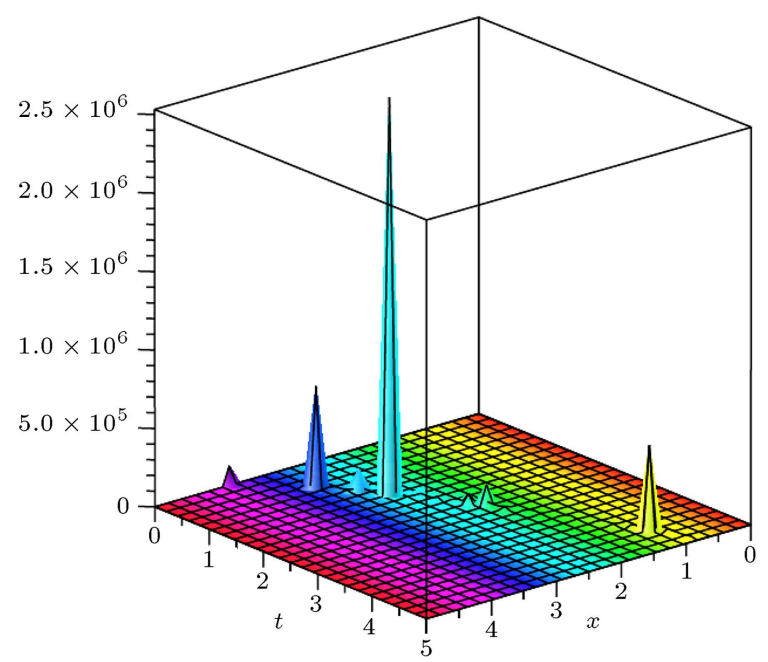

Figure 4. Graph of $u_{4}(x, y, t)$ of Eq. (21) corresponding to $\alpha=0.8$ for $k=0.85, c=0.5, l=0.5, \sigma=0.75, \mu=0.8$. $\delta=1.5, v=1.2$ and $\varepsilon=1.6$ at $y=0.75$.

of Eq. (21) corresponding to the values of $\alpha=1$ and $\alpha=0.8$ for $k=0.85, c=0.5, l=0.5, \sigma=0.75, \mu=$ $0.8, \delta=1.5, v=1.2$, and $\varepsilon=1.6$ at $y=0.75$.

\section{Conclusion}

In this article, a sub-equation method was successfully applied for solving one- and two-dimensional fractional RLW equations by using conformable fractional derivative. In this method, we have three types of exact solutions, two generalized hyperbolic function solutions, two generalized trigonometric function solutions, and one rational solution, which may be very useful to further understand the mechanisms of the complicated nonlinear physical phenomena and FPDEs. We can conclude that the proposed method can be extended to solve the nonlinear fractional problems which arise in the theory of solitons and other areas. Although this paper focuses just on the partial fractional differential equations with conformable fractional derivative, the fractional partial differential equations with beta derivative can be discussed based on the method of this paper. This will be the goal for investigation in the future works. The computations associated with the examples were performed using Maple 18.

\section{Acknowledgments}

We are very grateful to two anonymous referees for their careful reading and valuable comments which led to the improvement of this paper.

\section{References}

1. Ahmed, E. and Elgazzar, A.S. "On fractional order differential equations model for nonlocal epidemics", Phys. A., 379(2), pp. 607-614 (2007).

2. El-Sayed, A.M.A., El-Mesiry, A.E.M. and El-Saka, H.A.A. "On the fractional-order logistic equation", Appl. Math. Lett., 20(7), pp. 817-823 (2007).

3. Aminikhah, H., Refahi Sheikhani, A. and Rezazadeh, H. "Stability analysis of linear distributed order system with multiple time delays", U.P.B. Sci. Bull. Series A., 77(2), pp. 207-218 (2015).

4. Laskin, N. "Fractional market dynamics",Phys. A., 287, pp. 482-492 (2000).

5. Xin, B., Chen, T. and Liu, Y. "Projective synchronization of chaotic fractional-order energy resources demand-supply systems via linear control", Commun. Nonlinear Sci. Numer. Simulat., 16(11), pp. 4479-4486 (2011).

6. Podlubny, I. Fractional Differential Equations, Academic Press, San Diego (1999).

7. Jumarie, G. "Modified Riemann-Liouville derivative and fractional Taylor series of non-differentiable functions further results", Comput. Math. Appl., 51(9), pp. 1367-1376 (2006).

8. Liu, W. and Chen, K. "The functional variable method for finding exact solutions of some nonlinear timefractional differential equations", Pramana., 81(3), pp. 377-384 (2013).

9. Lu, B. "The first integral method for some time fractional differential equations", J. Math. Anal. Appl., 395(2), pp. 684-693 (2012).

10. Bin, Z. "Exp-function method for solving fractional partial differential equations", The Sci. World J., 2013, pp. 1-8 (2013).

11. Abdel-Salam, E.A.-B., Yousif, E.A., Arko, Y.A.S. and Gumma, E.A.E. "Solution of moving boundary spacetime fractional Burger's equation",J. Appl. Math., 2014, pp. 1-8 (2014). 
12. Abdel-Salam, E.A.-B. and Yousif, E.A. "Solution of nonlinear space-time fractional differential equations using the fractional Riccati expansion method", Math. Probl. Eng., 2013, pp. 1-6 (2013).

13. Abdel-Salam, E.A.-B. and Gumma, E.A.E. "Analytical solution of nonlinear space-time fractional differential equations using the improved fractional Riccati expansion method", Ain Shams Eng. J., 6(2), pp. 613$620(2015)$.

14. Abdel-Salam, E.A.-B. and Al-Muhiameed, Z.I.A. "Analytic solutions of the space-time fractional combined KdV-mKdV equation", Math. Probl. Eng., 2015, pp. 1-6 (2015).

15. Liu, C.S. "Counterexamples on Jumarie's two basic fractional calculus formulae", Commun. Nonlinear Sci. Numer. Simul., 22(1), pp. 92-94 (2015).

16. Khalil, R., Al Horani, M., Yousef, A. and Sababheh, M. "A new definition of fractional derivative", $J$. Comput. Appl. Math., 264, pp. 65-70 (2014).

17. Abdeljawad, T. "On conformable fractional calulus", J. Comput. Appl. Math., 279, pp. 57-66 (2015).

18. Atangana, A., and Doungmo Goufo, E.F. "Extension of matched asymptotic method to fractional boundary layers problems", Math. Probl. Eng., 2014, pp. 1-7 (2014).

19. Atangana, A., and Oukouomi Noutchie, S.C. "Model of break-bone fever via beta-derivatives", J. BioMed. Biotechnol., pp. 1-10 (2014). DOI 10.1155/2014/ 523159 .

20. Atangana, A. "A novel model for the lassa hemorrhagic fever: deathly disease for pregnant women", Neural. Comput. Appl. 26(8), pp. 1895-1903 (2015).

21. Zhang, S., Zhang, H.Q. "Fractional sub-equation method and its applications to nonlinear fractional
PDEs", Phys. Lett. A., 375(7), pp. 1069-1073 (2011).

22. Fan, E. "Tanh-function method and its applications to nonlinear equations extended", Phys. Lett. A., 277(4), pp. 212-218 (2000).

23. Benjamin, T.B., Bona, J.L. and Mahony, J.J. "Model equations for long waves in nonlinear dispersive systems", Philos. Trans. R. Soc. London A., 272(1220), pp. 47-78 (1972).

24. Huang, Z. "On Cauchy problems for the RLW equation in two space dimensions", Appl. Math. Mech., 23(2), pp. 159-164 (2002).

\section{Biographies}

Hossein Aminikhah was born in Iran, in 1979. $\mathrm{He}$ received a $\mathrm{PhD}$ degree in Applied Mathematics (Numerical Analysis) from University of Guilan, Rasht, Iran, in 2008, where he is currently Associate Professor. His research interests include numerical methods for functional differential equations and numerical linear algebra.

Amir Hossein Refahi Sheikhani was born in Iran, in 1980. He received a PhD degree in Applied Mathematics (Numerical Analysis) from University of Guilan, Rasht, Iran, in 2010. He is currently Assistant Professor in the faculty of Mathematical Sciences, Islamic Azad University of Lahijan. His research interests include numerical methods for PDEs and ODEs and fractional differential equations.

Hadi Rezazadeh is now a PhD student in the Applied Mathematics Department of University of Guilan. His interests and research areas include fractional differential equations. 